\title{
Increasing the efficiency of use of wheeled harrow units in regions of risk farming
}

\author{
A. E. Slepenkov", E. S. Polikutina, S. V. Shchitov, E. E. Kuznetsov, and Z. F. Krivutsa \\ Far Eastern State Agrarian University, Polytechnicheskaya street, 86, Blagoveshchensk
}

\begin{abstract}
Crop yields largely depend on the quality of the soil prepared for sowing. At the same time, one of the most important field operations is harrowing, with the main technological parameter of harrowing is the depth of processing of the fertile soil layer. Failure to do not have the necessary depth of processing leads to a decrease in yields, and the excess to additional energy costs, and therefore to increase the cost of a unit of the resulting products. As you know, the main parameter influencing the depth of processing is the weight that falls on the working organ of the harrow. This article proposes a device that has obtained a patent for the intellectual property of the Russian Federation, allowing to regulate the weight of the working organ of the harrow, due to its optimal redistribution within the machine-tractor unit (MTU), and the theoretical and experimental results of the experimental harrowing unit are provided. The results suggest that the proposed solution is highly effective, which provides as an opportunity for quality pre-planting treatment, and contributes to the compliance with the necessary agro-technological requirements for the harrowing process.
\end{abstract}

\section{Introduction}

The analysis of existing disc harrows has shown that currently produced both domestic and foreign designs are focused, as a rule, on light and medium low-clay soils. Moreover, if we analyze the main indicator that ensures the quality of field work, it is the depth of cultivation of the fertile layer. At the same time, this parameter directly depends on a number of indicators, including the vertical load on the working body (disk), its geometric parameters, as well as the position and spatial orientation on the field surface [1,2].

A number of scientific works are devoted to the issues of determining the basic geometric parameters of disk harrows [3,4]. The works of scientists are devoted to the substantiation of the relationship between the radius of curvature of the disk and its diameter.

In the studies, an analytical relationship was obtained between the diameter of the disc and the depth of cultivation with a harrow, which makes it possible to determine the required diameter of the working body (disc), which allows to optimize the depth of the cultivated soil layer. Perspective calculations of the parameters of spherical disks are given in the works of the authors $[5,6]$.

\footnotetext{
*Corresponding author: slepen555@mail.ru
} 
One of the problems when working with harrows with spherical disc working bodies is their sticking with soil at high humidity. To eliminate this problem, in study [4] it is proposed to use a spherical disc with slots. The author explains this by the fact that by reducing the area of contact with the soil, the specific pressure of the working body increases, thereby improving the process of its self-cleaning.

The quality of the disc harrows is largely determined by the rotational speed of the disc harrow. The author in [7] proposes to make asymmetric cuts for these purposes, which will ensure sliding cutting. To reduce clogging of discs with soil in study, it is proposed to ensure rotation of adjacent discs with different angular velocities.

At the same time, as shown by the analysis, all the proposed ways to improve the quality of harrowing work are aimed mainly at structures intended for work on sandy loam and chernozem soils with a low degree of moisture, which is not always acceptable for other soils.

From the above, it can be concluded that the vertical load on the working body is a fundamental parameter that ensures the burrowing capacity of the disc harrow. The analysis and review of existing designs of disc harrows showed that the vertical load on one disc ranges from $500 \ldots 600 \mathrm{H}$ to $1300 \ldots 1400 \mathrm{H}$, depending on the width and weight of the harrow, while they can be successfully operated on light and medium low-stone soils.

The land resources of the Amur Region are mainly represented by clay and heavy loamy soils with a maximum stickiness coefficient at high humidity. It should be noted that all work related to soil preparation for sowing and sowing in the region takes place in the presence of a solid underlying layer in the form of permafrost. At the same time, the climatic features of the region are such that, simultaneously with temperature fluctuations during the spring field work, uneven soil melting occurs, depending on the direction to the horizon and the slope of the terrain.

Thus, it can be concluded that in the conditions of the Amur Region it is necessary to use harrows that are capable of changing the value of the vertical load on the working body as quickly as possible, which will ensure the proper quality of the work performed.

In the studies carried out in $[8,9]$, it is noted that to ensure the optimal track depth, it is necessary to automatically reload the harrow working body due to the redistribution of weight in the links of the machine-tractor unit.

Consequently, the goal of the presented work is to create and test a reliable, highly efficient device with low cost, which allows to automatically adjust the load on the working body in order to improve the quality of soil preparation for sowing with the absolute fulfillment of agrotechnological requirements for machine and tractor units.

\section{Material and Methods}

As an example of the aforementioned device capable of automatically redistributing the adhesion weight as part of the MTA, it is proposed to use the adhesion weight regulator of the harrow unit for which RF patent No. 196181 was obtained [10].

For automatic adjustment of the tillage depth, it is proposed to install on the tractor a regulator of the coupling weight of the harrow unit, which allows it to maintain the required depth of tillage, depending on the agrotechnological requirements (Figure 1-2). 


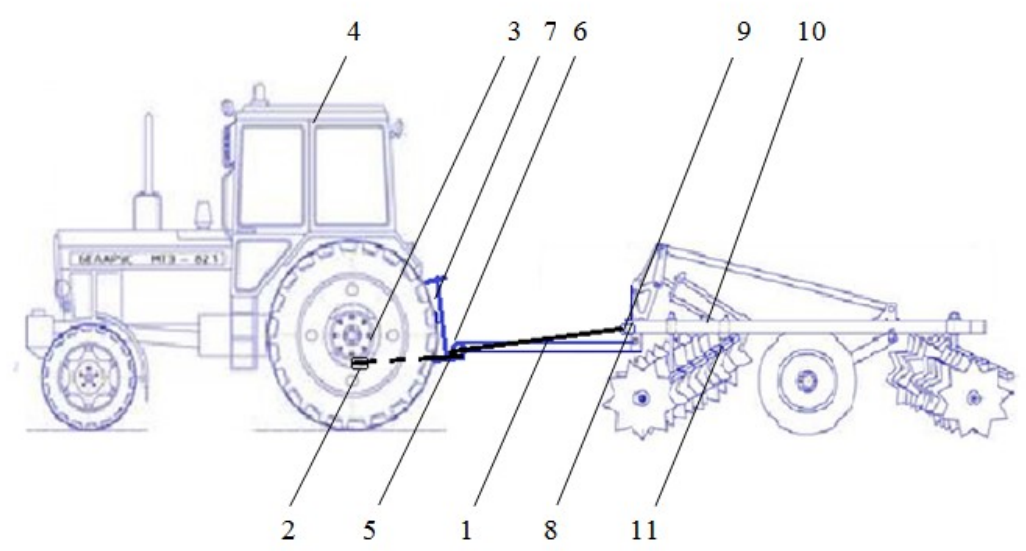

Fig. 1. Schematic diagram of the harrow unit coupling weight regulator:

1-cable power connection; 2-support bracket; 3- unit for installing a cable power connection; 4tractor body ; 5-towing device; 6-transverse lower link; 7-tractor hitch assemblies; 8-mount brackets; 8 - the cross beam of the harrow; 9 - harrow frame; 11 - disc harrow tools

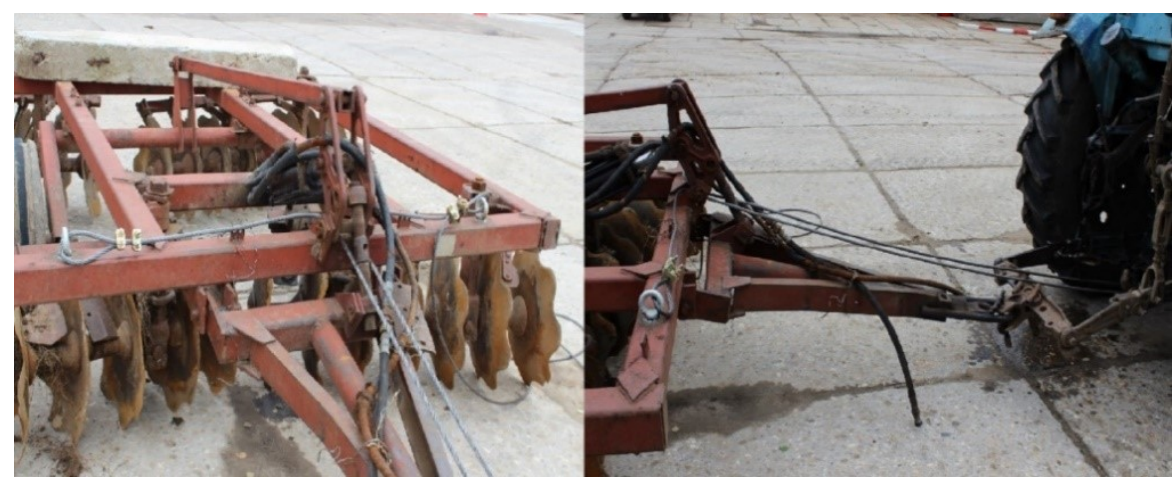

Fig. 2. General view of a tractor with a harrow unit coupling weight regulator

In more detail, the general structure and operation of the regulator of the adhesion weight of the harrow unit is given in study [11].

As an object for research on automatic adjustment of the working depth, a harrow unit was taken as a part of a tractor of class 1.4 of MTZ type and a heavy disc harrow BDT-3, on which a regulator of the adhesion weight of the harrow unit was installed.

Experimental studies were carried out according to the general and private methods recommended by the All-Russian Scientific Agroengineering Center (VIM-ARSAC). At the same time, an on-board measuring complex installed on a tractor, reading devices and devices, specialized programs for mathematical calculation, experiment modeling and wellknown methods of statistical and variance-regression analysis were used. During the experimental studies, the on-board measuring complex measured and recorded the following parameters: theoretical and operating speed, distance traveled, experience time, slipping, tractive effort, additional transmitted load. In the process of conducting experimental studies, the depth of processing by the disc working bodies of the harrow was also measured.

The processing of the data obtained during the experiment was carried out by the wellknown methods of mathematical statistics using information technologies [12]. 


\section{Results}

Conducted on the basis of the Far Eastern State Agrarian University, the city of Blagoveshchensk, the Amur region, the Russian Federation and production organizations of the region, theoretical and experimental studies on the use of the adhesion weight regulator of the harrow unit showed the validity and correctness of the chosen method of increasing the traction-coupling properties of the machine-tractor unit [6-11 ].

As a result of the theoretical studies, mathematical dependences were obtained, which made it possible to evaluate the effect of the regulator of the coupling weight of the harrow unit on the operating parameters of the harrow. The proposed device allows you to adjust the vertical load on the disk working body, thereby allowing you to adjust the depth of processing of the fertile layer. This issue is directly relevant for regions in which spring work takes place at a time when the depth of soil thawing in areas of the same arable field is different.

Let us consider the case when it becomes necessary to reduce the working depth with the harrow, using the working diagrams in Figures 3 and 4.

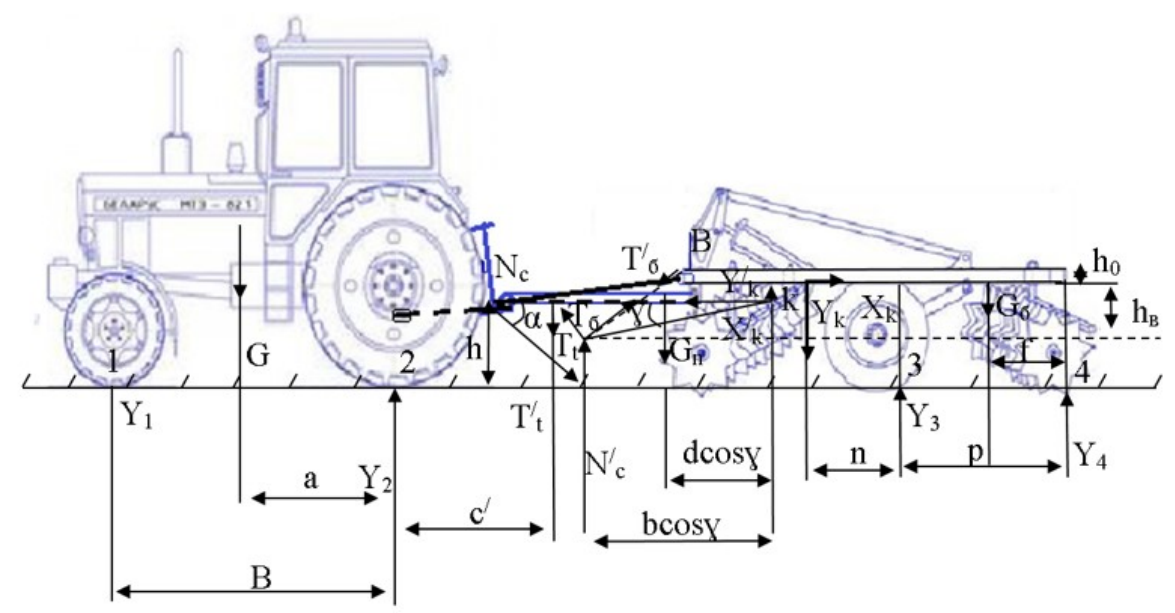

Fig. 3. Scheme for determining the vertical components of surface reactions during the operation of the adhesion weight regulator - lowering the hitch

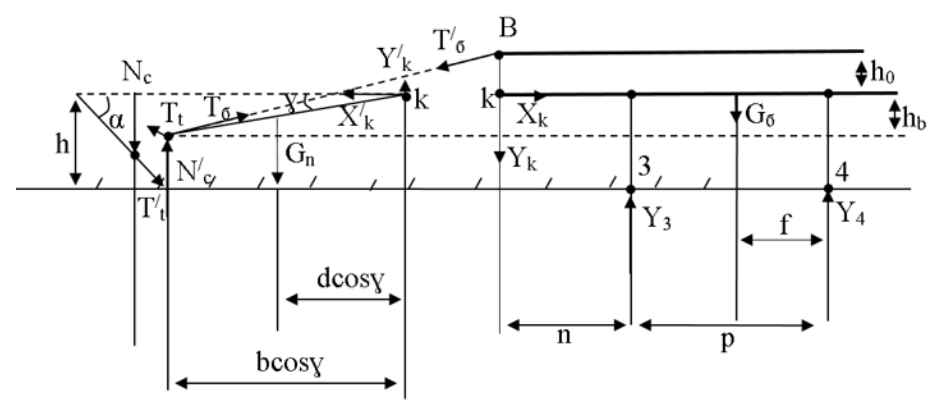

Fig. 4. Working scheme for determining and calculating the vertical components of the surface reactions during the operation of the adhesion weight regulator - lowering the hitch 
Where $G, G_{n}, G_{6}$ - is the weight of the tractor, hitch, harrow frame, respectively, $\mathrm{H}$; $\mathrm{Y}_{1}, \mathrm{Y}_{2}, \mathrm{Y}_{3}, \mathrm{Y}_{4}$ - force vertical reactions of the surface at the corresponding points, $\mathrm{H} ; \overline{\mathrm{N}_{\mathrm{c}}}=\overline{\mathrm{N}_{\mathrm{c}}^{\prime}}$ - force reaction of the internal connection at points $C, H ; \overline{X_{k}}=-\overline{X_{k}^{\prime}}$ and $\overline{Y_{k}}=-\overline{Y_{k}^{\prime}}$ - force reactions of the hinge at the point $\mathrm{K}, \mathrm{H} ; \mathrm{B}$ - longitudinal wheelbase of the tractor, $\mathrm{m}$; $\mathrm{a}$ distance from the center of gravity of the tractor to the rear propulsion support of the tractor, $\mathrm{m} ; \mathrm{c}$ - distance from the rear propulsion support of the tractor to point $\mathrm{C}, \mathrm{M} ; \mathrm{b}-$ the length of the hitch, $\mathrm{m} ; \mathrm{d}$ - distance from the center of gravity of the hitch to the hinge $\mathrm{K}, \mathrm{M}$; $p-$ is the distance from the points of application of vertical surface reactions to the harrow frame, $\mathrm{m} ; \mathrm{n}$ - is the distance from the hinge $\mathrm{K}$ to point 3 of the application of the vertical reaction of the surface to the section and frame of the harrow, $\mathrm{m} ; f$-Distance from the center of gravity of the harrow to the point of application of the vertical reaction of the surface to the section and frame of the harrow at point $4, \mathrm{M} . ; \mathrm{h}-$ Distance from the soil surface to the sample, m;

As a result of the theoretical studies, by constructing the equations of the equilibrium of the parts of the composite structure, a mathematical dependence was obtained, which makes it possible to determine the load on the working body of the harrow, depending on the design of the harrow unit and the features of the redistributing device, and to substantiate the parameters for expanding of its technological characteristics:

$$
\mathrm{Y}_{3}=\frac{\mathrm{G}_{\delta} \mathrm{f}}{\mathrm{P}}+\frac{\mathrm{G}_{\mathrm{n}}(\mathrm{B}-\mathrm{d})(\mathrm{n}+\mathrm{P})}{\mathrm{BP}}+\mathrm{F}\left(\begin{array}{c}
\frac{\cos \mathrm{d}}{\sin (\mathrm{d}+\theta)}- \\
-\frac{1}{\sin \theta+\cos \theta \operatorname{tg} \mathrm{d}}
\end{array}\right) \frac{\cos \theta \mathrm{h}_{\mathrm{B}}(\mathrm{n}+\mathrm{P})}{\mathrm{B} \cos \gamma \mathrm{P}}+\frac{\mathrm{F} \cos \theta \cos \mathrm{d}}{\sin (\mathrm{d}+\theta)} \cdot \frac{\mathrm{h}}{\mathrm{P}}+\frac{\mathrm{F}\left(\cos \theta \frac{\mathrm{h}_{0}}{\mathrm{P}}+\sin \theta\left(\frac{\mathrm{n}+\mathrm{p}}{\mathrm{P}}\right)\right.}{\sin \theta+\cos \theta \operatorname{tgd}} .
$$

Analyzing expressions (1), it should be noted that the proposed device allows you to increase the load on the harrow sections due to the use of a redistributing device.

To identify additional dependencies of the effect of the grip weight regulator of the harrow unit on the efficiency of the harrowing process and experimental confirmation of the theoretical results obtained, experimental studies were carried out in real operating conditions. The experiments were carried out in field production conditions, the soil background of which, according to its characteristics, corresponded to the agrotechnical requirements. In terms of its properties (mechanical composition), the soil was a heavy loam with a high degree of viscosity.

According to the obtained experimental data after their processing, the dependences presented in Figures 5 and 6 were built.

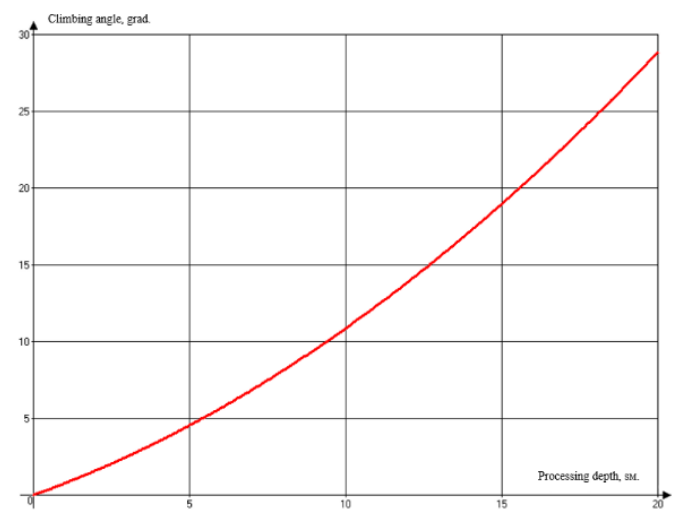

Fig. 5. Dependence of the tillage depth on the lifting angle of the device at the fixed values of the speed of the unit $(\mathrm{V}=7 \mathrm{~km} / \mathrm{h})$ and the width of the disc harrow $(\mathrm{b}=2.5 \mathrm{~m})$ (built on the basis of the data of the third factorial experiment) 
Analysis of the data obtained (Figure 5) allows us to conclude that with a decrease in the angle of lowering of the device, the depth of soil cultivation decreases.

During the operation of the harrow unit, it was found that the working depth also depends on the speed and width of the harrow unit. This relationship is visually visible on the research results presented in Figures 6 and 7.
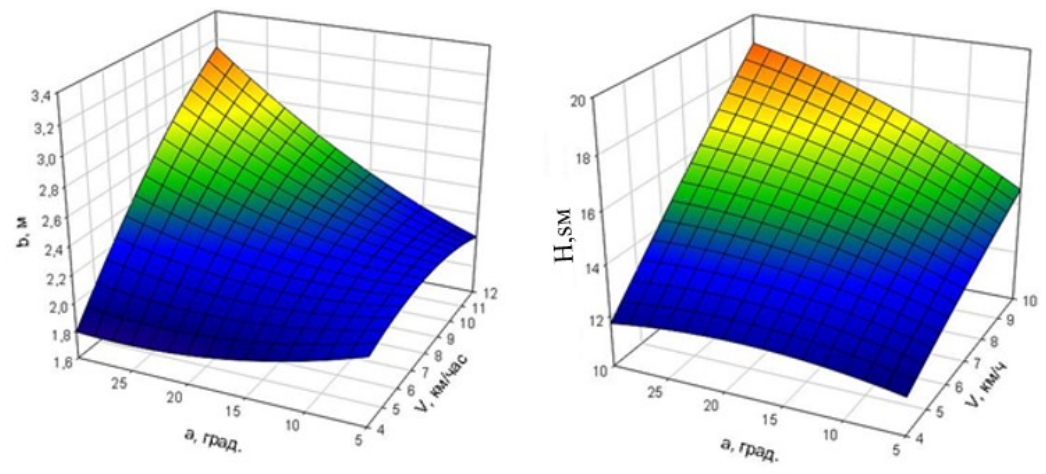

Fig. 6. Response surfaces (3D models) of the working depth and width of the disc harrow depending on the speed of the disc harrow and the lowering angle of the hitch (with $b=2.5$ meters and $h=16$ $\mathrm{cm}$ fixed at the zero level.)
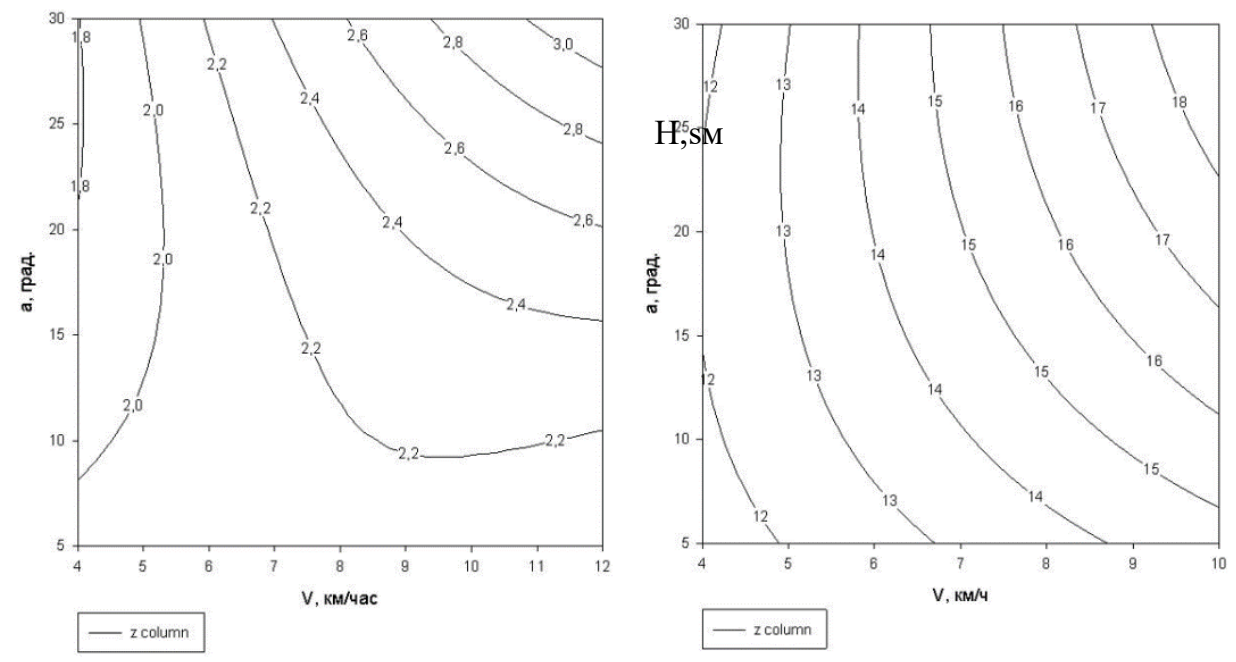

Fig. 7. Cross-section of the response surface (3D -model) of the dependence of the working depth and the width of the disc harrow on the speed of the disc harrow and the lowering angle of the traverse of the hitch (with $\mathrm{b}=2.5$ meters and $\mathrm{h}=16 \mathrm{~cm}$ fixed at the zero level.)

\section{Discussion}

Conducted theoretical and experimental studies have shown that the use of the proposed device allows you to adjust the depth of soil cultivation by regulating the load on the working body. 
With a maximum tillage depth of $20 \mathrm{~cm}$, the angle of the traverse of the tractor attachment to the device was 26-30 degrees, the width of the disc harrow was 2.2-2.8 meters, the speed of the unit was $9-10 \mathrm{~km} / \mathrm{h}$.

However, the results obtained in terms of comparing related indicators in previous studies [13-15] on this issue allow us to conclude that in the modern period of development of agricultural science, the proposed solution is the most rational, which undoubtedly distinguishes the proposed methodological and technical solutions, forming a new conceptual line in the environment of applied science, in particular, dedicated to the study of interaction in the field of knowledge "man-machine-nature" in agricultural production in the conditions of risky farming areas, dedicated to the study of improving the efficiency of soil preparation for sowing..

\section{Conclusion}

Based on the foregoing, it can be concluded that the proposed device, the regulator of the adhesion weight of the harrow unit, is a highly efficient design that implements the original ideas and has a design novelty intended for automatic regulation of the depth of soil cultivation. The research materials are used in the agricultural production technology used by «Soyuz» in the Oktyabrsky District and a number of other peasant farms in the Amur Region.

The introduction of the obtained results into production made it possible to increase the efficiency of the process of preparing the soil for sowing when using the harrow MTA in the technology of cultivating agricultural crops. Methodological and mathematical solutions are massively used in the educational process of the federal state budgetary educational institution of higher education "Far Eastern State Agrarian University".

\section{Reference}

1. M. G. Bekker, Introduction to Terrain-Vehicle Systems, 1, 520 (1973)

2. M. Behnassi, M. Syomiti Muteng'e, G. Ramachandran, K. N. Shelat, Vulnerability of Agriculture, Water and Fisheries to Climate Change: toward Sustainable Adaptation Strategies, 336 (2014)

3. D. Wulfsohn, T. R. Way, Advances in Soil Dynamics, 3, 209 (2019)

4. G.N. Sinyakov, I.M. Panov, Theory and calculation of tillage machines, 311 (1977)

5. J. Bulinski, H. Niemczyk, Ann.Warsaw Agr. Univ. Agr., 50, 3 (2007)

6. V.I. Belyaev, M. Fruhauf, T. Mainel, Europa Regional, 1(4), 13 (2004)

7. F.M. Kanarev, Rotary tillage machines and implements, 142 (1983)

8. E. E. Kuznetsov, The use of the multi-axis energy devices of class 1.4,153 (2013)

9. E.E. Kuznetsov, S.V. Shchitov, Improving the efficiency of the use of mobile energy resources in the technology of cultivation of agricultural crops, 272 (2017)

10. Shchitov S.V., Kuznetsov E.E., Pat. for utility model No. 196181 Rus. Federation applicant and patent holder Far East state. agr. university. declared 2019, registered 09.25.2019, 5, 10 (2019)

11. A.E. Slepenkov, O.A. Kuznetsova, Actual problems of science and technology, Issue VI, Samara, 21 (2019)

12. Milan Janić, Advanced Transport Systems: Analysis, Modeling, and Evaluation of Performances, 408 (2014) 
13. Keshav Maharjan, Joshi Lall, Prakash Niraj, Climate Change, Agriculture and Rural Livelihoods in Developing Countries: Series: Advances in Asian HumanEnvironmental Research, 176 (2013)

14. Armando Mendes, L. D. G. Soares da Silva, Azevedo Santos Emiliana, M Jorge, Efficiency Measures in the Agricultural Sector: With Applications, 197 (2013)

15. Eric Lichtfouse, Sustainable Agriculture Reviews, Series: Sustainable Agriculture Reviews, 15, 407 (2015) 